\title{
Liquid Cell Transmission Electron Microscopy for Electrochemical Processes
}

\author{
Frances M. Ross
}

IBM T. J. Watson Research Center, Yorktown Heights, NY, USA.

Liquid cell electron microscopy enables visualization of liquid phase processes with the high resolution of the transmission electron microscope $[1,2]$. Although the technique has been broadly applied for materials and biological imaging, it is particularly well suited for electrochemical studies because its combination of spatial and temporal resolution cannot easily be achieved using other techniques. Observing electrochemical reactions in situ requires integration of electrodes within the liquid cell and accurate control of the electrolyte geometry and the electrochemical parameters, voltage or current.

Here we describe the opportunities for liquid cell microscopy to help probe electrochemical processes. We first discuss experimental design. The electrode geometry must be chosen to ensure that the experiment replicates, to as high a degree as possible, the process taking place in a benchtop electrochemical cell. To test this, several types of calibration experiment prove useful. Figure 1 shows two examples for the case of copper deposition [3,4]. The images shown also illustrate two possible experimental geometries: plan view in (a), where a reaction is imaged on the surface of an electrontransparent electrode, and side view in (b), obtained when growth extends outwards from the edge of the electrode. The experimental geometry can be optimized for the process of interest, whether it is a corrosion reaction, growth front evolution, or additive effects on morphology, these latter two of particular interest for battery operation. It is also important to evaluate electron beam effects on the electrolyte [5, 6] and on the reaction pathway [e.g. 7]. Water radiolysis calculations show complex effects that include changes in $\mathrm{pH}$ towards lower values, the creation of bubbles of $\mathrm{H}_{2}$ or $\mathrm{O}_{2}$ and the formation of hydrated electrons that react with metal ions in solution to form metal nanocrystals $[5,6]$.

Additional phenomena can be explored by enhancing the functionality in the liquid cell. Figure 2 shows an example in which liquid flow is used to change the electrolyte chemistry. A galvanic displacement reaction is triggered by adding $\mathrm{Pd}^{3+}$ ions to an electrolyte flowing over $\mathrm{Ni}$, forming $\mathrm{Pd}$ particles on the electrode. This creates a nanopatterned surface with useful catalytic properties for subsequent electroless deposition. We find a latency time between changes in solution and the visible start of such reactions. Experiments involving a combination of corrosion and beam-induced precipitation [8] can provide information on diffusion processes within the thin liquid cell that may help explain such latency.

The continued development of liquid cell TEM will enable quantitative visualization of an even broader range of processes. Controlled mixing of liquid streams near the viewing area will yield more precise relationships between electrolyte composition and reaction kinetics. The integration of temperature control with electrochemical capabilities will open a new class of reactions for study. Compositional analysis is a new frontier for liquid cell microscopy that we expect will have a strong impact on electrochemical deposition experiments [2]. We therefore anticipate a promising future for liquid cell microscopy for the study of electrochemical processes [9].

\section{References:}

[1] N de Jonge and FM Ross, Nature Nanotechnology 6 (2011) p. 695. 
[2] FM Ross, Science 350 (2015), aaa9886.

[3] MJ Williamson et al, Nature Materials 2 (2003), p. 532.

[4] A Radisic et al, Nano Letters 6 (2006), p. 238.

[5] JM Grogan et al, Nano Letters 14 (2014), p. 359.

[6] NM Schneider et al, J Physical Chemistry C 118 (2014), p. 22373.

[7] M den Heijer et al, APL Materials 2 (2014), 022101.

[8] JH Park et al, Microscopy and Microanalysis 20 (3) (2014), p. 1598.

[9] We acknowledge J. H. Park, N. M. Schneider, M. C. Reuter and A. W. Ellis.
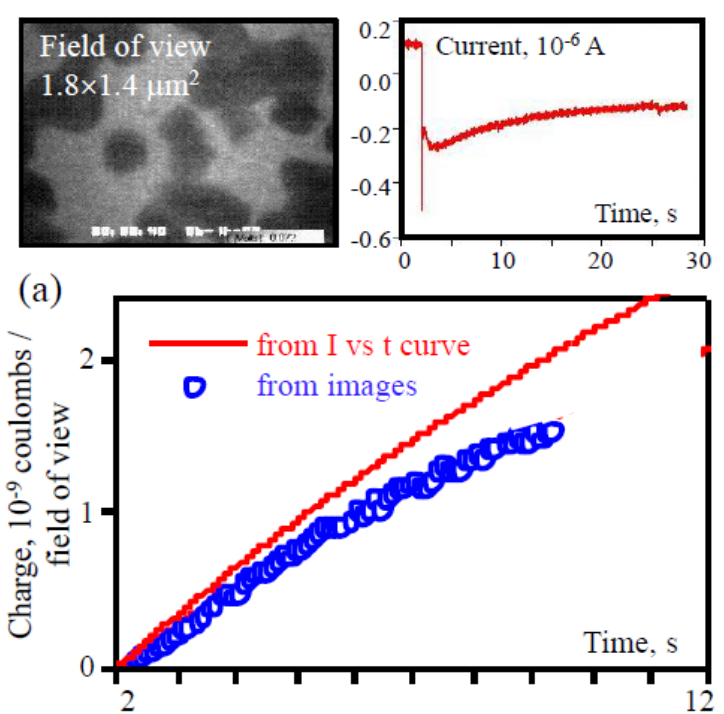

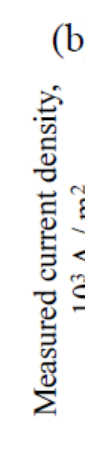

(b)
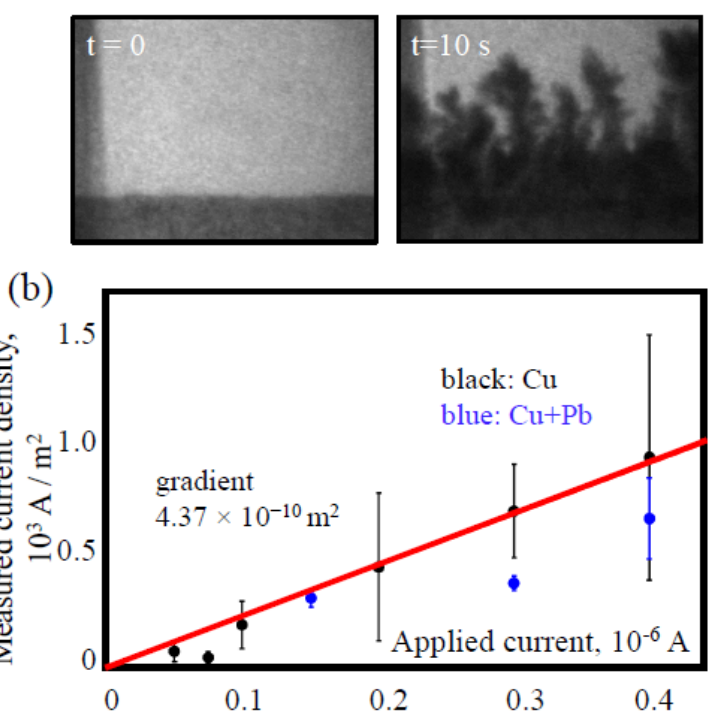

Figure 1. Calibration experiments for liquid cell TEM electrochemistry. (a) Movie frame and current flow for potentiostatic $\mathrm{Cu}$ deposition on $\mathrm{Au}$ from $0.1 \mathrm{M} \mathrm{CuSO}_{4}+0.18 \mathrm{M} \mathrm{H}_{2} \mathrm{SO}_{4}$ at $-0.07 \mathrm{~V}$ vs. a $\mathrm{Cu}$ pseudo-reference electrode. Below, circles show the charge passed through the field of view, estimated from the movie, assuming two electrons per deposited $\mathrm{Cu}$ atom and hemispherical $\mathrm{Cu}$ clusters (dark features in the image); line shows the charge passed, estimated from the current scaled by the ratio of the imaged area $\left(2.5 \times 10^{-12} \mathrm{~m}^{2}\right)$ to the total electrode area $\left(4.0 \times 10^{-9} \mathrm{~m}^{2}\right)$. The two estimates agree within $20 \%$, suggesting that the area imaged is representative of the entire electrode area. (b) Galvanostatic $\mathrm{Cu}$ deposition on $\mathrm{Pt}$ from the same electrolyte with and without $\mathrm{Pb}$ additive. Data points show the current density at the imaged area measured from the growth front propagation rate (images above) vs. the total current. The ratio gives the effective area over which current flows. This is similar to the area expected from the geometry of the liquid cell and electrode.
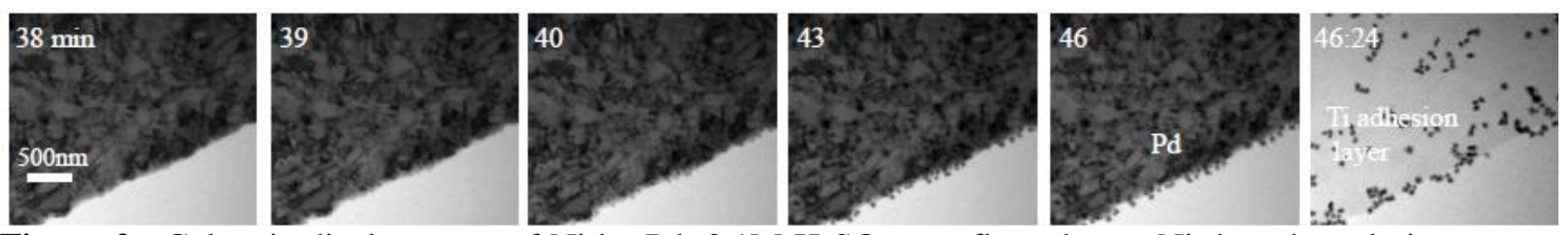

Figure 2. Galvanic displacement of $\mathrm{Ni}$ by $\mathrm{Pd} .0 .1 \mathrm{M} \mathrm{H}_{2} \mathrm{SO}_{4}$ was flowed over $\mathrm{Ni}$ then the solution was changed to add $\mathrm{PdSO}_{4}$. The movie shows that after some latency time Pd particles form on the $\mathrm{Ni}$, consistent with the expected reaction $2 \mathrm{Pd}^{3+}+3 \mathrm{Ni}^{0} \rightarrow 2 \mathrm{Pd}^{0}+3 \mathrm{Ni}^{2+}$. Within a few seconds all $\mathrm{Ni}$ is removed, partly due to the galvanic reaction and partly a radiolysis effect. 